\title{
Ajuste personal y conductas disruptivas en alumnado de primaria
}

\section{Personal Adjustment and Disruptive Behaviors in Primary School Students}

\author{
Marta Martínez-Vicente ${ }^{1}$ \\ (1) https://orcid.org/0000-0002-6601-9674 \\ Carlos Valiente-Barroso ${ }^{2}$ \\ (1D https://orcid.org/0000-0003-4670-0523
}

\author{
${ }^{1}$ Facultad de Humanidades y Ciencias Sociales, Universidad Internacional Isabel I, España \\ ${ }^{2}$ Departamento de Neurociencia, Instituto Clínico y de Investigación Interdisciplinar en
} Neurociencias, España

Resumen. Objetivo. Estudiar las relaciones entre variables de ajuste personal y las conductas disruptivas en un grupo de 136 alumnos y alumnas de primaria de entre 9 y 12 años. Método. Estudio de campo observacional, de metodología no experimental y transversal. Se utilizaron varios cuestionarios para medir la autoestima, la personalidad, el estrés y las competencias emocionales de los y las estudiantes, así como un cuestionario ad hoc elaborado para el registro de las conductas disruptivas. Resultados. Los resultados indican relaciones significativas positivas entre conductas disruptivas y estrés escolar, así como negativas con autoestima, estabilidad, competencia y comprensión emocional. Las diferencias son significativas según el género, manifestándose las conductas inadecuadas en menor medida en el caso de las niñas.

Palabras clave. Conductas disruptivas, estrés percibido, autoestima, competencia emocional, personalidad.

Abstract. Objective. This study analyses the relationships between personal adjustment and disruptive behaviors in a group of 136 primary school students between the ages of 9 and 12. Method. This field observation study used a non-experimental, cross-sectional methodology. Several questionnaires were used to measure students' selfesteem, personality, stress, and emotional competencies. Moreover, an ad hoc questionnaire was created to record disruptive behaviors. Results. The results indicate significant positive relationships between disruptive behaviors and school stress, as well as negative ones with self-esteem, stability, competence, and emotional understanding. The differences are significant according to gender, with inappropriate behaviors manifesting to a lesser extent in the case of girls.

Keywords. Disruptive behaviors, Perceived stress, Self-esteem, Emotional Competency, Personality.

'Marta Martínez-Vicente. Facultad de Humanidades y Ciencias Sociales, Universidad Internacional Isabel I. Dirección postal: Hermandad de Donantes de Sangre, 1, 3 B, 39200,España. E-mail:mmv3619@hotmail.com

${ }^{2}$ Carlos Valiente-Barroso. Departamento de Neurociencia, Instituto Clínico y de Investigación Interdisciplinar en Neurociencias, España. E-mail: carlosvbsiete@hotmail.com

\section{(C) $(\mathcal{D} \Theta \Theta$}




\section{Introducción}

Las conductas disruptivas son comportamientos que entorpecen el correcto funcionamiento del aula. Se consideran como tales, la violación de las normas, la alteración en el trabajo escolar, la oposición a la autoridad docente, las agresiones verbales o físicas a otros compañeros; pero también otras menos graves como hacer ruido, levantarse, interrumpir, hablar con frecuencia, ser irresponsable o mostrar desinterés y desmotivación. Suponen un inconveniente para el docente, los compañeros y el propio estudiante, y junto a otros problemas socioeducativos y el bajo rendimiento escolar, provocan una constante desmotivación en el profesorado (Mora \& Ponce, 2017). El malestar docente ha aumentado como resultado de la propagación de contextos de aula donde imperan conductas distorsionantes, falta de disciplina y una tensión atribuida a estudiantes denominados disruptivos, que impiden con sus comportamientos y actitudes el normal funcionamiento de las clases.

Delimitar o definir una conducta disruptiva no es tarea fácil pues depende entre otros aspectos del nivel de tolerancia, la interpretación, la experiencia personal, la percepción y las estrategias de control y manejo del aula de cada docente. En general, una conducta inadecuada es aquella que atenta contra los principios educativos impidiendo la marcha normal de la clase. Sus causas son diversas y se deben en parte a la carencia de estrategias de afrontamiento que desencadenan problemas diarios, malestar emocional y conductas de desobediencia (Bueno, 2014; Porcel, 2010). El aula es el lugar en el que se produce una mayor cantidad de conductas agresivas dada la heterogeneidad del alumnado y sus marcadas diferencias individuales, acentuándose en aquellas que reciben a estudiantes de diferentes edades, algo que, si bien en principio puede ser un elemento enriquecedor, también puede comportar muchos inconvenientes (Martín \& Lleixá, 2017).

Las conductas disruptivas se categorizan de mayor o menor gravedad según las relaciones interpersonales, las tareas escolares y las normas generales del aula. Se asocian a factores como la impulsividad, escasas habilidades sociales, relaciones interpersonales negativas, poca capacidad de autocrítica, falta de autoestima, dificultad para cumplir las normas, baja tolerancia a la frustración, escasas estrategias de afrontamiento del estrés y bajo rendimiento académico. Causas que responden a la respuesta insuficiente ofrecida en el ámbito escolar, a las que además se añaden los estilos parentales en la educación y la ausencia de relaciones seguras y afectivas dentro del ámbito familiar (Díaz-Aguado et al., 2004; Gotzens et al., 2010).

Los problemas de disciplina suponen una preocupación frecuente en el ámbito educativo limitando tanto el tiempo de docencia del profesorado como de aprendizaje del alumnado. La resolución de conflictos implica dedicar gran parte del horario escolar a solventarlos, con lo que resta para otro tipo de actividades más placenteras y estimulantes. El mal ambiente generado dentro de algunas aulas, el desorden o la elevada incidencia de problemas comportamentales, reclama actuaciones preventivas y específicas que resuelvan tales realidades. Algunos autores distinguen entre dos tipos de conductas disruptivas: las de índole social que alteran la dinámica y el orden de las aulas (Ramírez \& Justicia, 2006) y las instruccionales, derivadas del desconocimiento de las normas que regulan el funcionamiento del aula (Grebennikov, 2005). Tanto unas como otras dependen de la gestión docente, ya sea en la prevención o en la intervención directa cuando así es necesario (Gotzens et al., 2007). 
Actualmente algunos comportamientos agresivos y el incremento de los niveles de victimización física y verbal se justifican por el uso excesivo de las TIC (Chacón-Cuberos et al., 2018). Sea por una causa u otra, la violencia se ha convertido en un fenómeno expansivo en los centros escolares a nivel internacional (Chacón-Cuberos et al., 2015; Méndez \& Cerezo, 2010). Dentro de las aulas se producen actuaciones transgresoras e intencionadas, conductas violentas provocadas por agresiones en las relaciones cuya finalidad es dañar a otros estudiantes (Carrasco et al., 2015; Olweus \& Limber, 2010; Zurita et al., 2015). Esta problemática preocupa tanto a nivel educativo como social pues provoca entre otras consecuencias, ansiedad, baja autoestima, depresión, estrés crónico, adicción a sustancias y bajo rendimiento académico (Chacón-Cuberos et al., 2015; Méndez \& Cerezo, 2010; Schneider et al., 2012). Al unísono, estos estados personales y psicológicos afectan a las relaciones interpersonales y la socialización provocando graves secuelas emocionales en los estudiantes que se perciben como centro de los ataques por parte de los iguales (Espejo et al., 2016; Estévez et al., 2012). Los niños y las niñas que manifiestan conductas disruptivas tienen dificultades para describir estados emocionales propios de las interacciones sociales (Ison, 2004) y estas conductas asociadas a la inestabilidad personal, impulsividad, pobre inteligencia emocional y baja tolerancia a la frustración, son potenciales factores de riesgo de los problemas de convivencia en las aulas (Murray et al., 2012). Así queda reflejado en estudios recientes que exploran la relación entre conductas disruptivas o violentas y las competencias sociomocionales, concluyendo que una mejora de estas últimas favorece las relaciones interpersonales y por ende un clima escolar positivo (Anderson \& Hunter, 2012; Diazgranados, 2014; Drerup \& Jackson, 2014; Gutiérrez-Cobo et al., 2017; Romero et al., 2017).

El ajuste personal es un constructo multidimensional que engloba tanto variables emocionales como psicológicas y que se evalúa a partir del clima o la satisfacción escolar, el rendimiento académico, los problemas externalizantes como el acoso, el abandono escolar y el apoyo social; y otros internalizantes como la depresión, el estrés o la soledad (Gutiérrez \& Gonçalves, 2013; Heras \& Navarro, 2012). Desde una aproximación holística del ajuste se han destacado el bienestar psicológico, el rendimiento escolar, las percepciones y el grado de implicación personal como indicadores de adaptación al contexto educativo y de garantía de éxito sociopersonal del alumnado (Ladd et al., 2011). A pesar de que aún son escasos los estudios sobre el tema, se informa de asociaciones significativas en el rendimiento académico y el autoconcepto social, el apoyo percibido por el grupo de iguales y las conductas prosociales (Valdés et al., 2012). Por lo tanto, las variables psicológicas inherentes a las características personales y contextuales repercuten facilitando o interfiriendo el óptimo desarrollo personal, social y académico de los y las estudiantes (Bisquerra et al., 2015; Usán \& Bordás, 2018).

Las competencias emocionales se han convertido en variables explicativas del desarrollo integral de los estudiantes dentro del contexto educativo. La conciencia y la comprensión emocional se cultivan a través de la inteligencia emocional y acentúan las actitudes empáticas y sociales (Carmona \& López, 2015; Gutiérrez \& López, 2015). Se han encontrado mejores conductas prosociales, un mayor bienestar personal y disminución de conductas disruptivas en estudiantes que han participado en programas para el fomento de las competencias emocionales (Filella-Guiu et al., 2014; Morente et al., 2017).

Las personas con niveles de satisfacción personal altos manifiestan rasgos importantes de ajuste psicosocial, siendo en el caso de los estudiantes esenciales en el clima relacional escolar, sobre todo en los casos de violencia directa o indirecta (Cava et al., 2010; 
Rodríguez-Fernández et al., 2012). Se han encontrado relaciones significativas negativas entre el desajuste escolar y clínico y síntomas emocionales, asociándose el sufrimiento de experiencias interpersonales negativas con déficits emocionales en la infancia (Dodge et al., 2003; Escobar et al., 2010).

La dimensión afectiva incluye aspectos como ser estable o inestable, tranquilo o inquieto, equilibrado o desequilibrado, tímido o asertivo. Dentro de ella la autoestima ocupa un lugar preponderante en la investigación dentro de la Psicología educativa, dada la importancia que tienen en la persona los sentimientos percibidos de sí misma (Rosenberg, 1965) que se manifiestan en la adaptación a eventos estresantes de la vida cotidiana y que junto con las competencias emocionales repercuten en el bienestar, la satisfacción e incluso la salud mental (Schoeps et al., 2019). Estudiantes con baja autoestima desvelan descontento, actitudes inseguras, desconfianza y demandan constantemente la atención. Una actitud asociada a la baja autoestima es la conducta desafiante y agresiva que exhiben estudiantes frustrados que compensan la falta de aprobación y su inseguridad con comportamientos agresivos. Las personas con autoestima alta controlan su vida, mientras que aquellas con valores bajos rigen sus comportamientos por la duda, el miedo y la defensión, siendo un elemento trascendental en la mitigación del estrés, así como en las acciones requeridas para su afrontamiento (Álvarez-Icaza et al., 2004). Las personas con autoestima alta tienen mayores posibilidades de anticipar ciertas situaciones, reaccionando según su propio juicio y afrontando los problemas, convirtiéndose en una pieza clave para mitigar el estrés (Jenkins \& Smith, 1990). La construcción de una autoestima óptima en la primera infancia constituye un modo de prevención en sí misma, revelándose pieza clave entre lo que el individuo responde ante sí mismo y el mundo externo. Los niños y niñas erigen su individualidad y cuando mantienen relaciones interpersonales adecuadas disponen de una fuerza personal adicional para rechazar situaciones inaceptables de intimidación o victimización (Robichaud, 2003).

En sinergia con la autoestima, el estrés percibido es diferente según los grupos de riesgo, y ambas variables son decisivas en el bienestar psicosocial de la persona, dependiendo a su vez directamente de la capacidad de afrontamiento. Según Lazarus y Folkman (1986) la situación concreta no es el elemento desencadenante del estrés, sino la valoración propia que la persona hace de la misma y en la que subyace la resistencia y el afrontamiento, factores determinantes para conseguir dicho bienestar. Un mismo acontecimiento se percibe de manera diferente por cada persona y desarrollan menos estrés aquellas que disponen de suficientes recursos para superarlo, así la percepción del estrés se convierte en predictora de una mayor vulnerabilidad en los grupos de riesgo (Cava et al., 2001).

Atendiendo al género, las niñas informan de niveles elevados de desajuste emocional impactando más en sus relaciones interpersonales al utilizarlas como recurso de afrontamiento (Seiffge-Krenke \& Stemmler, 2002). Además, ellas empoderan la inclusión social y la pertenencia a redes interpersonales afectivas, que cuando es de baja aceptación produce efectos negativos; lo que desarrolla una sintomatología internalizada como depresión y ansiedad (Reijntjes et al., 2006; Rudolph, 2002). Son las niñas las que informan de un mayor impacto emocional y mayores niveles de estrés percibido derivados de las relaciones interpersonales (Hampel \& Petermann, 2006; Rudolph, 2002). En contraposición, los niños tienden a manifestar otro tipo de conductas más agresivas (Escobar et al., 2010) y dentro de las estrategias de afrontamiento suelen emplear aquellas orientadas al problema, mientras que ellas usan estrategias como recurrir a los demás. Por ello, es importante disponer de un repertorio de estrategias de afrontamiento 
para un adecuado desarrollo psicopatológico, ya que cuando son improductivas o no constructivas repercuten directamente en relaciones sociales insatisfactorias (Reijntjes et al., 2006; Sandstrom, 2004).

En resumen, algunas conductas violentas y agresivas entre el estudiantado se convierten en un obstáculo para el desarrollo personal, social y escolar, al responder a previas provocaciones o al deseo de conseguir determinados beneficios por refuerzos externos, como por ejemplo querer ser líder en el grupo. El estrés psicológico, la depresión y las actitudes negativas hacia la escuela aumentan con los comportamientos agresivos ya sean físicos o verbales (Zimmer-Gembeck \& Pronk, 2012). Se consideran factores de riesgo generadores de estos patrones conductuales, la baja satisfacción hacia la vida (Martínez et al., 2012), el déficit empático (Evans et al., 2002), las relaciones negativas con los iguales (Murray \& Murray, 2004), elevados niveles de distrés psicológico (Villareal-González et al., 2011), la actitud negativa hacia los estudios (Estévez et al., 2013), la baja implicación académica (Álvarez-García et al., 2010) y una baja autoestima (Martínez et al., 2009).

Atendiendo a lo expuesto este trabajo se propone como objetivo analizar las relaciones entre las conductas disruptivas percibidas dentro del aula y variables de ajuste personal como son la autoestima, la personalidad, las competencias emocionales, el estrés infantil. Se espera encontrar diferencias significativas en todas las variables según el género, así como demostrar el carácter predictivo del ajuste personal en el desarrollo de las conductas disruptivas.

\section{Método}

Se plantea un estudio de campo observacional, de metodología no experimental, transversal, descriptivo y correlacional.

\section{Muestra}

Muestra no probabilística de conveniencia heterogénea. Participaron 136 estudiantes de Educación Primaria, 65 niños (47.79\%) y 71 niñas (52.20\%) de edades entre los 9 y 13 años $(M=9.75, D T=1.495)$ de nivel socioeconómico y cultural medio escolarizados en un centro concertado (de titularidad privada pero sostenido con fondos públicos) de una línea, situado en una localidad urbana de la comunidad autónoma de Cantabria (España).

\section{Instrumentos}

Cuestionario para la Evaluación de la Autoestima en Educación Primaria (AEP) (Ramos et al., 2006). Este instrumento compuesto de 17 ítems de respuesta tipo Likert de 3 puntos (0-Si, 1-A veces y $2-\mathrm{No}$ ) aporta una medida global de la autoestima. En este cuestionario se obtiene $\alpha=.76$.

Cuestionario de Personalidad para niños (CPQ) (Porter \& Cattell, 2016). Evalúa 14 rasgos de primer orden y 3 de segundo orden de la personalidad. Los rasgos de primer orden son afabilidad (reservado/abierto), razonamiento (inteligencia baja/alta), estabilidad emocional (afectado/estable), excitabilidad (calmoso/estable), dominancia (sumiso/dominante), animación (sobrio/entusiasta), atención a las normas (despreocupado/consciente), atrevimiento (cohibido/emprendedor), sensibilidad (dura/blanda), desadaptación (seguro/dubitativo), privacidad (sencillo/astuto), aprensión (sereno/aprensivo), perfeccionismo (menos/más integrado) y tensión (relajado/tenso). A partir de las escalas primarias se obtienen tres factores de segundo orden globales sobre ajuste/ansiedad, introversión/ extraversión y patemia/excitabilidad-dureza ( $\alpha=.81)$. 
Inventario de Estrés Cotidiano Infantil (IECI) (Trianes et al., 2011). Consta de 22 ítems dicotómicos (SI/NO) que proporcionan puntuaciones parciales correspondientes a tres ámbitos del estrés infantil (salud, escolar y familiar), además de una medida de estrés global resultado de la suma de las tres.Para la escala estrés total se obtiene $\alpha=.81$.

Cuestionario de competencia emocional para niños TEIQue-CSF de Petrides (Adaptación española de Pérez-González, 2003), considerada como una de las medidas evaluativas de la inteligencia emocional más válida y fiable. Se compone de 36 ítems de respuesta tipo Likert $(0=$ Completamente en desacuerdo; 4 = Completamente de acuerdo) que evalúan habilidades emocionales. Para este estudio se obtiene $\alpha=.764$.

Cuestionario de comprensión emocional para niños KAI-R-VP -Forma corta para profesores- (Kusche et al., 1988. Adaptación española de Pérez-González, 2003) que recoge información del profesor sobre la comprensión de las competencias emocionales de los estudiantes. Consta de seis ítems de respuesta tipo Likert $(0=$ Completamente en desacuerdo; 4 = Completamente de acuerdo). Para este estudio se obtiene $\alpha=.931$.

Cuestionario ad hoc sobre conductas disruptivas en el aula elaborado para este estudio. Se trata de un registro observacional para que el profesor registre las conductas disruptivas que interfieren en el normal desarrollo de las clases. Consta de 28 ítems de respuesta tipo Likert 4 puntos ( $1=$ Nunca, $2=A$ veces, $3=$ Muchas veces y $4=$ Siempre). Para este estudio se obtiene $\alpha=.982$.

\section{Procedimiento}

El trabajo fue puesto en conocimiento del Comité de Ética del Instituto Clínico y de Investigación Interdisciplinar en Neurociencias (ICIIN) para su envío posterior. Una vez autorizado se informó a los tutores y familias contando con la aprobación expresa a través de un documento firmado por padres, madres o tutores legales, cumpliéndose de esta manera las exigencias deontológicas indicadas para investigación con menores de edad. Los cuestionarios fueron aplicados en una sesión dentro del horario lectivo por parte del equipo investigador, siempre en presencia del tutor o tutora. Se informó a los y las estudiantes de la finalidad del estudio, solicitando su participación voluntaria y sinceridad en las respuestas, garantizando en todo momento el anonimato y la confidencialidad de los datos obtenidos. Asimismo, los cuestionarios ad hoc sobre conductas disruptivas y el cuestionario de comprensión emocional KAI-R-VP de cada uno de los alumnos y alumnas fueron cumplimentados por los tutores de las aulas a las que pertenecía cada uno de los y las estudiantes participantes.

\section{Análisis de datos}

Técnicas estadísticas descriptivas, $t$ de Student y análisis de varianza unifactorial (ANOVA) para estudiar si existen diferencias significativas y comparar puntuaciones medias de las variables según el género, midiendo el tamaño del efecto a través del coeficiente eta-cuadrado parcial $\left(\eta_{p}^{2}\right)$ (efecto pequeño si $\eta_{p}^{2} \geq .01$, efecto medio si $\eta_{p}^{2}$ $\geq .059$ y efecto grande si $\eta_{p}^{2} \geq$.138). Se calcula también la $d$ de Cohen para conocer la magnitud de las diferencias (Sun et al., 2010) interpretándose como tamaño del efecto pequeño $(.20 \leq d \leq .50)$, moderado $(.51 \leq d \leq .79)$ y grande $(d \geq .80)$. Se calculan correlaciones de Pearson para estudiar las relaciones entre las variables y un análisis de regresión múltiple (Stepwise) para estudiar el carácter predictivo de todas ellas sobre las conductas disruptivas. Todos los cálculos se realizaron con el programa estadístico SPSS versión 25.0 para Windows. 


\section{Resultados}

\section{Análisis descriptivo y diferencial}

Teniendo en cuenta el objetivo general se realiza un análisis descriptivo de los ítems del cuestionario de conductas disruptivas (Figura 1) que informa de valores elevados en comportamientos como la despreocupación ante las tareas, hablar mucho en clase, falta de conocimientos y de hábitos de estudio, irresponsabilidad, pasividad, jugar dentro de clase, desinterés y atención dispersa.

Se estudiaron las diferencias de medias de las variables según el género a partir del cálculo de la $t$ de Student, aplicando la prueba de Levene para la igualdad de varianzas a través del estadístico $F$, considerando niveles de significación mayores de .05 y un intervalo de confianza del $95 \%$. Los resultados indican diferencias significativas en autoestima, comprensión emocional, estabilidad emocional, sensibilidad, dureza y conductas disruptivas según el género (Tabla 1).

El análisis de varianza (ANOVA) indica que las niñas puntúan más alto en autoestima, comprensión emocional, estabilidad emocional y sensibilidad, mientras que los niños lo hacen en patemia/excitabilidad-dureza y conductas disruptivas dentro del aula, siendo el tamaño del efecto grande en todas estas variables (Tabla 2).

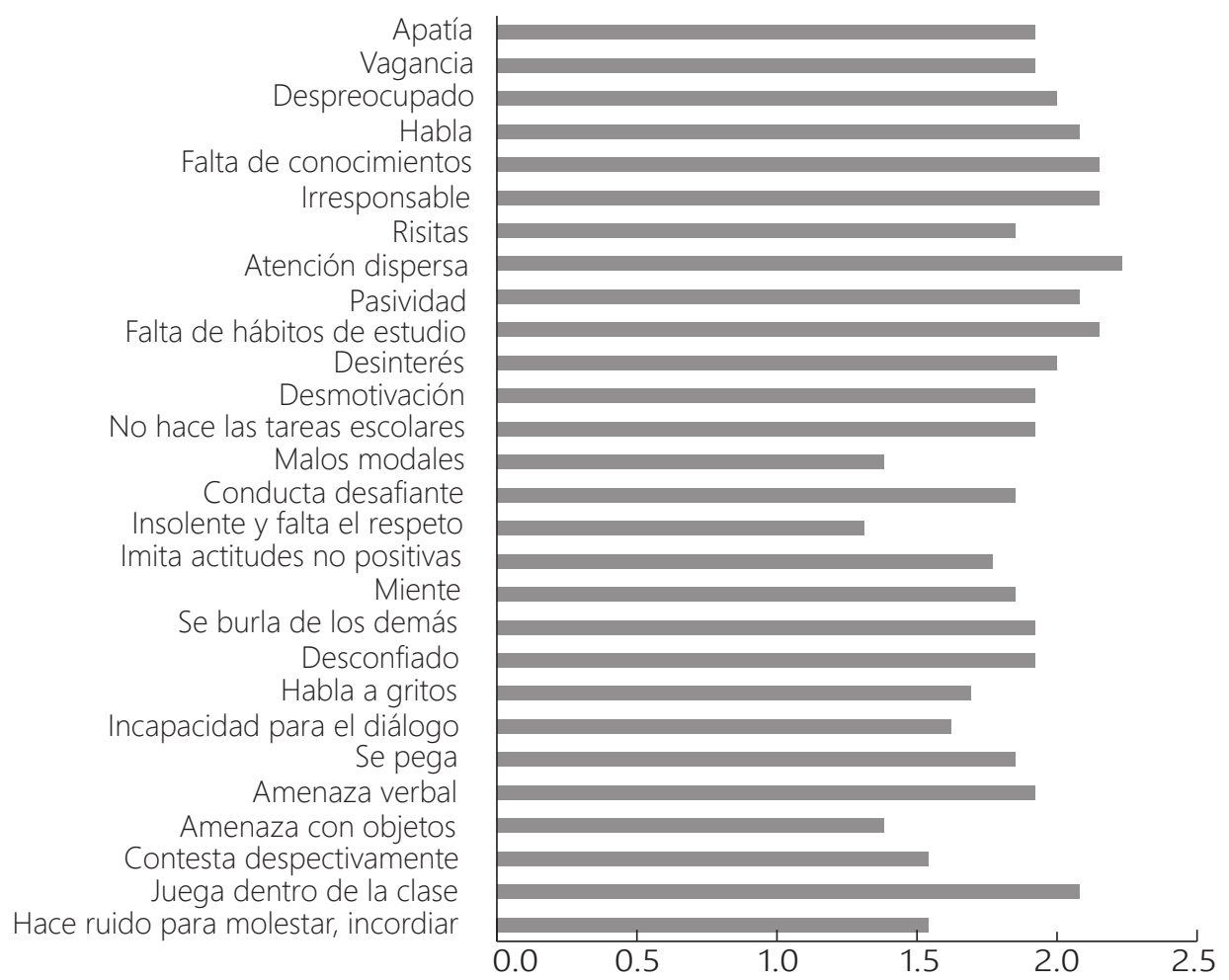

Figura 1. Conductas disruptivas valoradas en el cuestionario 
Tabla 1

Resultados de la prueba t para muestras independientes en función del género

\begin{tabular}{|c|c|c|c|c|c|c|c|c|}
\hline & \multicolumn{2}{|c|}{$\begin{array}{c}\text { Prueba de } \\
\text { Levene }\end{array}$} & \multicolumn{6}{|c|}{ Prueba $t$ para la igualdad de medias } \\
\hline & \multirow[t]{2}{*}{$F$} & \multirow[t]{2}{*}{ Sig. } & \multirow[t]{2}{*}{$t$} & \multirow{2}{*}{$\begin{array}{c}\text { Sig. } \\
\text { Bilateral }\end{array}$} & \multirow{2}{*}{$\begin{array}{l}\text { Diferencia } \\
\text { de medias }\end{array}$} & \multirow{2}{*}{$\begin{array}{l}\text { Error típico de } \\
\text { la diferencia }\end{array}$} & \multicolumn{2}{|c|}{ IC al $95 \%$} \\
\hline & & & & & & & $\mathrm{LI}$ & LS \\
\hline Autoestima & .899 & .363 & -2.095 & $.046^{*}$ & -22.357 & 10.670 & -45.84 & 1.13 \\
\hline $\begin{array}{c}\text { Comprensión } \\
\text { emocional }\end{array}$ & .250 & .627 & -2.864 & $.015^{\star}$ & -6.000 & 2.095 & -10.61 & -1.39 \\
\hline $\begin{array}{l}\text { Estabilidad } \\
\text { emocional }\end{array}$ & .542 & .477 & -2.679 & $.021^{*}$ & -2.048 & .764 & -3.73 & -.36 \\
\hline Sensibilidad & 4.080 & .068 & -2.016 & $.049 *$ & -2.405 & 1.193 & -5.03 & .22 \\
\hline $\begin{array}{c}\text { Patemia/ } \\
\text { excitabilidad- } \\
\text { dureza }\end{array}$ & 2.256 & .161 & 2.226 & $.048 *$ & 1.721 & .773 & .02 & 3.42 \\
\hline $\begin{array}{l}\text { Conductas } \\
\text { disruptivas }\end{array}$ & .052 & .823 & 5.437 & $.000^{\star *}$ & 37.119 & 6.826 & 22.09 & 52.14 \\
\hline
\end{tabular}

Nota. Solo aparecen las variables con diferencias significativas en función del género. $F=$ estadístico $F$ de la prueba de Levene; $t=$ índice $t$ de Student; Sig = nivel de significación; $I C=$ intervalo de confianza; $\mathrm{LI}=$ límite inferior; $\mathrm{LS}=$ límite superior.

${ }^{*} p<.05 ;{ }^{*} p>.01$

Tabla 2

Medias (Desviaciones típicas) y análisis de varianza de las variables en función del género

\begin{tabular}{|c|c|c|c|c|c|c|c|}
\hline \multirow[t]{2}{*}{ Variables } & \multirow[t]{2}{*}{ Grupos } & \multirow[t]{2}{*}{$M(D T)$} & \multicolumn{2}{|c|}{ IC al $95 \%$} & \multirow[t]{2}{*}{$F(1,11)$} & \multirow{2}{*}{$\eta_{p}^{2}$} & \multirow[t]{2}{*}{$d$} \\
\hline & & & $\mathrm{LI}$ & LS & & & \\
\hline \multirow[t]{2}{*}{ Autoestima } & Chico & $27.50(16.051)$ & 10.66 & 44.34 & 4.390 & .285 & -1.180 \\
\hline & Chica & 49.86(21.443) & 30.03 & 69.69 & & & \\
\hline \multirow{2}{*}{$\begin{array}{c}\text { Comprensión } \\
\text { emocional }\end{array}$} & Chico & $9.00(4.00)$ & 4.80 & 13.20 & 8.201 & .427 & -1.584 \\
\hline & Chica & $15.00(3.559)$ & 11.71 & 18.29 & & & \\
\hline \multirow{2}{*}{$\begin{array}{l}\text { Estabilidad } \\
\text { emocional }\end{array}$} & Chico & $3.67(0.816)$ & 2.81 & 4.52 & 7.177 & .395 & -1.527 \\
\hline & Chica & $5.71(1.704)$ & 4.14 & 7.29 & & & \\
\hline \multirow[t]{2}{*}{ Sensibilidad } & Chico & $6.17(2.927)$ & 3.10 & 9.24 & 4.066 & .270 & -1.081 \\
\hline & Chica & $8.57(1.134)$ & 7.52 & 9.62 & & & \\
\hline \multirow{2}{*}{$\begin{array}{c}\text { Patemia/ } \\
\text { excitabilidad- } \\
\text { dureza }\end{array}$} & Chico & $3.95(1.834)$ & 2.03 & 5.87 & 4.956 & .311 & 1.200 \\
\hline & Chica & $2.23(0.860)$ & 1.43 & 3.02 & & & \\
\hline \multirow{2}{*}{$\begin{array}{l}\text { Conductas } \\
\text { disruptivas }\end{array}$} & Chico & 71.83(13.526) & 57.64 & 86.03 & 29.564 & .729 & 2.998 \\
\hline & Chica & 34.71(11.116) & 24.43 & 44.99 & & & \\
\hline
\end{tabular}

${ }^{*} p<.05 ;{ }^{* *} p<.01$ 


\section{Análisis correlacional}

Se realiza un análisis correlacional considerando las variables autoestima, estrés, competencia emocional, comprensión emocional y conductas disruptivas (Tabla 3). Los resultados indican relaciones significativamente positivas entre las variables autoestima y comprensión emocional; estrés escolar con estrés salud, estrés global y conductas disruptivas; estrés salud con estrés global y competencia emocional y esta última con comprensión emocional. Asimismo, se observan relaciones significativas negativas entre autoestima con estrés familiar y conductas disruptivas; estrés escolar y comprensión emocional; así como competencia y comprensión emocional con conductas disruptivas.

Un segundo análisis correlacional entre los factores de personalidad y las variables autoestima, estrés, competencia emocional, comprensión emocional y conductas disruptivas arroja los resultados de la Tabla 4. Se observan relaciones significativamente positivas entre autoestima y estabilidad emocional e integración; estrés escolar y desadaptación; estrés familiar y excitabilidad; estrés salud y estrés global con excitabilidad y desadaptación; competencia y comprensión emocional con estabilidad emocional y por último entre conductas disruptivas y patemia/excitabilidad-dureza. Asimismo, se relacionan negativamente autoestima y desadaptación, ajuste/ansiedad y patemia/ excitabilidad-dureza; estrés escolar con razonamiento y estabilidad emocional; estrés familiare introversión/extraversión; estrés salud y razonamiento; estrés global con razonamiento, perfeccionismo e introversión/extraversión y por último entre conductas disruptivas y estabilidad emocional.

Tabla 3

Correlaciones entre autoestima, estrés, competencia emocional, comprensión emocional y conductas disruptivas

\begin{tabular}{lcccccccc}
\hline & 1 & 2 & 3 & 4 & 5 & 6 & 7 & 8 \\
\hline 1. Autoestima & 1 & & & & & & & \\
2. Estrés escolar & -.298 & 1 & & & & & & \\
3. Estrés familiar & $-.576^{\star}$ & .010 & 1 & & & & & \\
4. Estrés salud & -.020 & $.580^{\star}$ & .250 & 1 & & & & \\
5. Estrés global & -.411 & $.686^{\star *}$ & .589 & $.861^{\star *}$ & 1 & & & \\
6. Competencia emocional & .171 & .538 & -.253 & $.558^{\star}$ & .412 & & & \\
7. Comprensión emocional & $.649^{\star}$ & $-.646^{\star}$ & .277 & .175 & .015 & $.817^{\star \star}$ & 1 & \\
8. Conductas disruptivas & $-.624^{\star}$ & $.570^{\star}$ & .450 & .689 & .401 & $-.768^{\star \star}$ & $-.717^{\star \star}$ & 1 \\
\hline
\end{tabular}

${ }^{*} p<.05 ;{ }^{* *} p<.01$ 
Tabla 4

Correlaciones entre los factores de personalidad y autoestima, estrés, competencia emocional, comprensión emocional y conductas disruptivas

\begin{tabular}{|c|c|c|c|c|c|c|c|c|}
\hline & $\mathrm{AU}$ & $\mathrm{EE}$ & $\mathrm{EF}$ & ES & EG & CE & CPE & $C D$ \\
\hline$A$ & .243 & .130 & -.062 & -.020 & -.080 & .094 & .115 & -.172 \\
\hline B & .351 & $-.622^{*}$ & -.191 & $-.607^{\star}$ & $-.686^{\star \star}$ & .342 & .364 & -.340 \\
\hline C & $.594^{*}$ & $-.585^{\star}$ & .012 & -.143 & -.341 & $.878^{* *}$ & $.825^{\star *}$ & $-.853^{* *}$ \\
\hline D & -.509 & .263 & $.614^{\star}$ & $.586^{\star}$ & $.750^{\star *}$ & -.115 & .014 & .343 \\
\hline$E$ & -.051 & .157 & .479 & .113 & .353 & .168 & .287 & .021 \\
\hline $\mathrm{F}$ & -.246 & .018 & .026 & -.203 & -.111 & -.151 & -.441 & .271 \\
\hline G & .503 & -.036 & -.340 & -.153 & -.324 & .180 & .225 & -.398 \\
\hline $\mathrm{H}$ & .388 & -.036 & -.176 & .063 & -.130 & .408 & .252 & -.352 \\
\hline I & .544 & -.186 & -.365 & .183 & -.182 & .350 & .434 & -.535 \\
\hline J & $-.563^{*}$ & $.596^{\star}$ & .457 & $.752^{\star \star}$ & $.855^{\star \star}$ & -.313 & -.096 & .323 \\
\hline$N$ & -.303 & .278 & .428 & .263 & .446 & -.098 & .047 & .357 \\
\hline O & -.517 & .040 & .511 & .348 & .496 & -.185 & .001 & .262 \\
\hline $\mathrm{Q}_{3}$ & $.616^{\star}$ & -.339 & -.434 & -.324 & $-.589 *$ & .324 & .365 & -.546 \\
\hline $\mathrm{Q}_{4}$ & -.179 & .018 & .507 & .393 & .493 & .266 & .366 & -.211 \\
\hline $\mathrm{Q}_{1}$ & $-.560 *$ & .196 & .352 & .352 & .508 & -.344 & -.260 & .461 \\
\hline $\mathrm{Q}_{\|}$ & .475 & -.235 & $-.578^{*}$ & -.464 & $-.664^{*}$ & .075 & -.040 & -.289 \\
\hline $\mathrm{Q}_{\text {III }}$ & $-.608^{*}$ & .297 & .513 & .065 & .441 & -.377 & -.400 & $.648^{*}$ \\
\hline
\end{tabular}

Nota. $\mathrm{AU}=$ Autoestima; $\mathrm{EE}=$ Estrés escolar; $\mathrm{EF}=$ Estrés familiar; $\mathrm{ES}=$ Estrés salud; $\mathrm{EG}=$ Estrés global; $C E=$ Competencia emocional; $C P E=$ Comprensión emocional; $C D=$ Conductas disruptivas; $\mathrm{A}=$ Afabilidad (reservado/abierto); $\mathrm{B}=$ Razonamiento (inteligencia baja/alta); $\mathrm{C}=$ Estabilidad emocional (afectado/estable); D = Excitabilidad (calmoso/excitable, $\mathrm{E}=$ Dominancia (sumiso/ dominante); $F=$ Animación (sobrio/entusiasta); $G=$ Atención a las normas (despreocupado/ consciente); $\mathrm{H}=$ Atrevimiento (cohibido/emprendedor); $\mathrm{I}=$ Sensibilidad (dura/blanda); $\mathrm{J}=$ Desadaptación (seguro/dubitativo); $\mathrm{N}=$ Privacidad (sencillo/astuto); $\mathrm{O}=$ Aprensión (sereno/ aprensivo); $\mathrm{Q}_{3}=$ Perfeccionismo (menos/más integrado); $\mathrm{Q}_{4}=$ Tensión (relajado/tenso); $\mathrm{Q}_{1}=$ Ajuste/Ansiedad, $\mathrm{Q}_{\|}=$Introversión/Extraversión; $\mathrm{Q}_{\| \mid}=$Patemia/excitabilidad-dureza.

${ }^{*} p<.05 ;{ }^{* *} p<.01$

\section{Análisis de regresión}

Finalmente, se realizó un análisis de regresión múltiple (procedimiento stepwise) para saber qué variables predicen las conductas disruptivas, indicando los resultados que son tres variables del total (Tabla 5). Así, en el modelo 1 se observa que la variable género explica el $70.4 \%$ de la varianza total, siendo su capacidad predictiva sobre las conductas disruptivas ( $\beta=-.854 ; t=$ $-5.437: p<.001)$ estadísticamente significativa. En el modelo 2 se incluyen las variables género $(\beta$ $=-.774 ; t=-7.722 ; p<.001)$ y estrés escolar $(\beta=.425 ; t=4.242 ; p<.05)$ explicando juntas el 88.4 $\%$ de la varianza, contribuyendo significativamente a la explicación de las conductas disruptivas dentro del aula. En el modelo 3, junto a las anteriores, se incluye la variable estabilidad emocional, explicando conjuntamente el $92 \%$ de la varianza total y tanto género $(\beta=-.607 ; t=-5.531 ; p<$ $.001)$, estrés escolar $(\beta=.274 ; t=2.606 ; p<.05)$ y estabilidad emocional $(\beta=-.311 ; t=-2.343 ; p$ $<.05)$ explican significativamente las conductas disruptivas. Del modelo quedaron excluidas el resto de las variables que formaban parte de este estudio. 
Tabla 5

Resultados del análisis de regresión con conductas disruptivas como variable criterio y las variables vinculadas del estudio como variables predictoras

\begin{tabular}{lccccc}
\hline Modelo & $R$ & $R^{2}$ & $R^{2}$ ajustado & $F(\mathrm{gl})$ & $p$ \\
\hline Modelo $1^{\mathrm{a}}$ & .854 & .729 & .704 & $29.564(1,12)$ & .000 \\
Modelo $^{\mathrm{b}}$ & .950 & .903 & .884 & $46.617(2,12)$ & .000 \\
Modelo $^{\mathrm{c}}$ & .969 & .940 & .920 & $46.853(3,12)$ & .000 \\
\hline
\end{tabular}

aGénero. 'bénero, Estrés escolar. 'Género, Estrés escolar, Emocionalmente estable/afectado

\section{Discusión}

Durante los últimos años se ha incrementado la tendencia al estudio de todos los factores que afectan al desarrollo personal, emocional y social de los y las estudiantes; y que pueden ser el sustrato sobre el que se asientan determinados comportamientos o reacciones desproporcionadas visibles en algunos momentos. En concreto, se señalan las conductas disruptivas como un elemento de preocupación en el ámbito educativo intentando profundizar en qué puede provocar que determinados estudiantes interfieran o dificulten la actuación docente o las relaciones del grupo en el que están escolarizados. Por ello en el presente trabajo se describe el estudio llevado a cabo en un centro educativo de ratio reducida en el que se observan y registran por parte de los tutores de aula conductas de tipología disruptiva del alumnado, para posteriormente compararla con la información recogida sobre variables de índole personal a través de la cumplimentación de cuestionarios por los propios niños y niñas y con todo ello ofrecer una explicación de por qué las conductas disruptivas dificultan la convivencia.

En primer lugar, se extrajo la información recogida en el cuestionario ad hoc sobre conductas disruptivas cuyos resultados informan de que la despreocupación en las tareas, hablar interrumpiendo constantemente al profesor, la irresponsabilidad, la carencia de conocimientos básicos y de hábitos de estudio, la pasividad, el desinterés hacia el trabajo y la atención dispersa son, en general, algunas de las conductas disruptivas más frecuentes dentro de las aulas.

La comparativa de las variables consideradas teniendo en cuento al género, permite observar cómo las niñas puntúan más alto en autoestima, comprensión emocional, estabilidad emocional y sensibilidad, presentando los niños una mayor dureza y también un mayor número de las conductas disruptivas analizadas. En esta línea se encuentran algunos estudios que informan de que las conductas agresivas y disruptivas son exhibidas principalmente por los niños, quienes, a pesar de integrarse fácilmente, también son objetivo directo del fenómeno del acoso (Escobar et al., 2010; Lippman \& Rivers, 2008; Ros et al., 2012).

Por otro lado, los resultados del análisis correlacional han mostrado asociaciones directas entre la autoestima y la competencia y estabilidad emocional, al tiempo que estas son inversas con las conductas disruptivas. Estas conductas presentan asociaciones directas con el estrés estudiantil coincidiendo con una mayor dureza e inestabilidad emocional. En general, este estudio está en consonancia con otros previos que confirman que la concepción positiva de sí mismo se corresponde con un mejor ajuste psicológico, una adecuada competencia personal y menos problemas comportamentales (Fuentes et al., 
2011; Pérez-Escoda et al., 2014). Así, se justifica la importancia de considerar el impacto que tiene el estado emocional negativo en el desarrollo de problemáticas de mayor gravedad (Filella-Guiu et al., 2014) y cómo fomentar conductas positivas, la regulación emocional y actitudes positivas reducen la vulnerabilidad, el estrés y el desarrollo de comportamientos distorsionadores o contaminantes del clima escolar (Matthews et al., 2012; Morente et al., 2017).

Los resultados hallados en este trabajo seguirían la línea marcada por otros que evalúan la influencia de la autoestima en la percepción y el afrontamiento del estrés (Álvarez-Icaza et al., 2004). Estudios que confirman que una autoestima alta influye en el control personal para afrontar directamente los problemas, aumentando la confianza de los estudiantes en su resolución y mejorando su adaptación a nivel escolar, familiar y social. Sin embargo, cuando la autoestima es baja, el comportamiento se rige por sentimientos de duda, miedo y actitudes de defensa (Field, 1993), pudiendo ser la explicación de que dicha persona actúe según los demás y no de ella misma. Así se caracterizan los estudiantes con un autoconcepto bajo, deficientes relaciones familiares y sociales, con dificultades de adaptación escolar, distrés y problemas de afrontamiento que intentan resolver sus problemas a través de otras personas (Millon, 1998). Por lo tanto, el equilibrio emocional depende de la gestión exitosa del afrontamiento y de las habilidades desarrolladas para tolerar o manejar las situaciones generadoras de estrés.

Por último, el análisis de regresión múltiple efectuado confirma el carácter predictivo del estrés escolar, el componente emocional y el género en las conductas disruptivas de los estudiantes de primaria. Es posible que si niveles bajos de autoestima interfieren en el desarrollo emocional de los estudiantes provoquen estados de estrés y ansiedad asociados directamente con comportamientos inadecuados fruto de tales carencias. Estos resultados tienen similitud con el estudio de Estévez y Jiménez (2015), en el que de manera recíproca se demuestra que el comportamiento agresivo hacia los iguales, tanto en niños como niñas, predice positiva y significativamente, distintos indicadores tanto de ajuste escolar como personal. Se relacionan significativamente el estrés percibido, la sintomatología depresiva y los sentimientos de soledad con menores puntuaciones en autoestima, satisfacción con la vida y empatía. La agresión predice bajas puntuaciones en actitud positiva hacia la escuela y el profesorado, implicación académica, apoyo percibido por el profesor y afiliación con los compañeros. Resultados que sugieren que las conductas disruptivas y agresivas con los iguales pueden reducir los niveles de satisfacción con la vida y la empatía, siendo predictoras de la autoestima (Estévez \& Jiménez, 2015). La asimetría de las relaciones entre los estudiantes influye en las interacciones necesarias para el desarrollo personal en el periodo escolar y las conductas agresivas afectan a los sentimientos de afiliación y de amistad entre compañeros, lo que al mismo tiempo repercute en el rendimiento escolar. Por otro lado, no hay que olvidar que la infancia constituye un periodo clave en el desarrollo vital, durante la que se construye la personalidad y se cultivan las competencias emocionales, forjándose los valores y los comportamientos prosociales en cada niño y niña (Gutiérrez, 2020).

Una de las limitaciones de este estudio deriva de la revisión de los trabajos realizados hasta el momento, siendo aún escasos los que relacionan conductas disruptivas y agresivas con factores de ajuste personal como el estrés o la autoestima en población adolescente, y menos aún en la infancia. Además, la mayor parte de los estudios se centran por un lado en variables de ajuste personal como la autoestima, y por otro, en el ámbito comportamental (conducta antisocial), sin estudiar la sinergia entre dicho 
conjunto de variables. Otra limitación es el tamaño muestral, los estudiantes pertenecen a un centro de una línea (ratio reducida), no permitiendo generalizar los resultados obtenidos. Además, se indica como limitación el carácter transversal y correlacional del estudio, que impide sacar conclusiones más allá del momento en el que se recogen los datos y no es posible establecer relaciones causa-efecto, por lo que se proponen estudios longitudinales para conocer si las diferencias individuales y las conductas disruptivas se mantienen a lo largo de la escolaridad obligatoria, siendo necesario extender la muestra a las etapas de Secundaria y Bachillerato.

Atendiendo a lo expuesto, la etapa de Educación Primaria se configura como la más idónea para adquirir las competencias socioemocionales que desde la escuela deben favorecer la adaptación a un entorno en constante cambio. Como precursora del desarrollo máximo de los estudiantes, se enfrenta a un amplio abanico heterogéneo tanto en lo que se refiere a capacidades como a necesidades personales y debe proporcionar diferentes oportunidades y experiencias de aprendizaje significativas. Actualmente, corrientes como la Psicología Positiva permiten comprender el ajuste personal como resultado de la interacción alumno-escuela-familia, dejando atrás el modelo centrado en el déficit, significativo del siglo pasado, que dificultaba una interpretación positiva del desarrollo de competencias promotoras de la adaptación al entorno y el bienestar personal (Agirre et al., 2015). El aula es el lugar donde mayor número de transacciones interpersonales se producen y se comparten conductas que en ocasiones originan situaciones de elevada conflictividad. La detección temprana puede incrementar la efectividad en la intervención ante determinadas conductas, facilitando el proceso de enseñanza-aprendizaje, algo en lo que es clave la actuación e implicación directa del docente (Gómez \& da Resurrección, 2017; Rodríguez, 2011).

Es preciso añadir en futuras investigaciones otros indicadores como la empatía o la satisfacción vital, para clarificar la aportación de todas estas variables de bienestar en el ajuste personal en las etapas tanto de Primaria como de Secundaria (Agirre et al., 2015). El estudio de las competencias emocionales puede ser el camino para una adecuada comprensión de las diferencias individuales ante la exposición al riesgo, aportando datos significativos en lo que se refiere a la conflictividad presente en las aulas (Estévez \& Jiménez, 2015).

Por otro lado, es crucial la actuación y coordinación docente en la intervención ante las conductas disruptivas. Si bien no existe un estilo docente mejor que otro, y sí diferentes estilos de comunicación e interacción, se disponen de estrategias de eficacia demostrada como por ejemplo los refuerzos positivos y la autorreflexión grupal (Latorre \& Teruel, 2009). Deben facilitarse al alumnado herramientas que faciliten el cambio de los hábitos disruptivos por habilidades participativas y de cooperación (Alonso \& Juste, 2008), siendo un cauce en la gestión de las conductas disruptivas la disciplina asertiva con autoridad, sin olvidar las circunstancias personales de cada alumno.

Como conclusión final, se enfatiza la necesidad de que cada centro diseñe programas adaptados a sus necesidades para trabajar las habilidades socioemocionales con actividades centradas en la autoestima, la empatía, la asertividad y las habilidades de interacción y de autoafirmación; y cuyo fin último sea mejorar el autocontrol, prevenir la violencia, proporcionar estrategias adecuadas de resolución de problemas, desarrollar un autoconcepto positivo y mejorar la competencia social incrementando la tolerancia y el respeto a la diversidad. 


\section{Referencias}

Agirre, I. A., Izaguirre, L. A., Etxaniz, I. E., \& Maya, M. S. (2015). Capacidad predictiva del autoconcepto y la inteligencia emocional en el ajuste escolar autopercibido. Bordón. Revista de Pedagogía, 67(4), 9-26. https://doi.org/10.13042/ Bordon.2015.67401

Alonso, J. D., \& Juste, M. R. P. (2008). Las conductas problemáticas en el aula: propuesta de actuación. Revista Complutense de Educación, 19(2), 447-457. https://dx.doi. org/10.5209/RCED

Álvarez-García, D., Álvarez, L., Núñez, J. C., González, P., González-Pienda, J. A., Rodríguez, C., \& Cerezo, R. (2010). Violencia en los centros educativos y fracaso académico. Revista Iberoamericana de Psicología y Salud, 1(2), 139-153. https://www.redalyc. org/articulo.oa?id=245116406002

Álvarez-Icaza, M. A. V., Gómez-Maqueo, E. L., \& Durán-Patiño, C. (2004). La influencia de la autoestima en la percepción del estrés y el afrontamiento en niños de edad escolar. Salud Mental, 27(4), 18-25. https://www.redalyc.org/pdf/582/58242703.pdf

Gutiérrez, A. (2020). Inteligencia emocional percibida en estudiantes de educación superior: análisis de las diferencias en las distintas dimensiones. Actualidades en Psicología, 34(128), 17-33. https://doi.org/10.15517/ap.v34i128.34469

Anderson, S., \& Hunter, S. C. (2012). Cognitive appraisals, emotional reactions, and their associations with three forms of peer-victimization. Psicothema, 24(4), 621-627.

Bisquerra, R., Pérez, J. C., \& García, E. (2015). Inteligencia emocional en educación. Editorial Síntesis.

Bueno, N. (2014). Afrontament de conductes disruptives en l'àmbit escolar. Educat: Revista de Psicopedagogía, 3, 11-12. https://www.educat.cat/blog/wp-content/ uploads/2014/01/conductes-disruptives.pdf

Carmona, M. G., \& López, J. E. (2015). Autoconcepto, dificultades interpersonales, habilidades sociales y conductas asertivas en adolescentes. Revista Española de Orientación y Psicopedagogía, 26(2), 42-58. https://doi.org/10.5944/reop.vol.26. num.2.2015.15215

Carrasco, C., Alarcón, R., \& Trianes, M. (2015). Eficacia de una intervención psicoeducativa basada en clima social, violencia percibida y sociométricos en alumnado de Educación Primara. Revista Psicodidáctica, 20(2), 247-262. https://doi.org/10.1387/ RevPsicodidact.13206.

Cava, M. J., Arango, C. M., \& Musitu, G. (2001). Autoestima, percepción de estrés y ánimo depresivo en grupos de riesgo. Cuadernos de Trabajo Social, 14(17), 17-28. https:// revistas.ucm.es/index.php/CUTS/article/view/CUTS0101110017A

Cava, M. J., Buelga, S., Musitu, G., \& Murgui, S. (2010). Violencia escolar entre adolescentes y sus implicaciones en el ajuste psicosocial: un estudio longitudinal. Revista de Psicodidáctica, 15(1), 21-34.

Chacón-Cuberos, R., Espejo-Garcés, T., Martínez-Martínez, A., Zurita-Ortega, F., CastroSánchez, M., \& Ruiz, G. R. R. (2018). Conductas agresivas, victimización y uso problemático de videojuegos en escolares de educación primaria de la provincia 
de Granada. Revista Complutense de Educación, 29, 1011-1023. http://dx.doi. org/10.5209/RCED.54455

Chacón-Cuberos, R., Martínez-Martínez, A., Castro-Sánchez, M., Espejo-Garcés, T., ValdiviaMoral, P. A., \& Zurita-Ortega, F. (2015). Relación entre bullying, género y actividad física: Estudio en escolares de la provincia de Granada. TRANCES, 7(6), 791-810.

Díaz-Aguado, M. J., Martínez-Arias, R., \& Martín-Seoane, G. (2004). La violencia entre iguales en la escuela y en el ocio. Estudios comparativos e instrumentos de evaluación. Instituto de la Juventud.

Diazgranados, S. (2014). Asociación entre los ambientes escolares y las actitudes de apoyo hacia la violencia en estudiantes colombianos. Revista Colombiana de Educación, $66,175-202$.

Dodge, K. A., Lansford, J. E., Burks, V. S., Bates, J. E., Pettit, G. S., Fontaine, R., \& Price, J.M (2003). Peer rejection and social information-processing factors in the development of aggressive behavior problems in children. Child Development, 74, 374-393. https:// doi.org/10.1111/1467-8624.7402004

Drerup. L., \& Jackson, Y. (2014). Community Violence and Psychological Adjustment in Youth: Role of emotional-social intelligence. Journal of Child and Adolescent Trauma, 7(1), 17-26. https://doi.org/10.1007/s40653-014-0006-z

Escobar, M., Trianes, M. V., Fernández-Baena, F. J., \& Páez, J. M. (2010). Relaciones entre aceptación sociométrica escolar e inadaptación socioemocional, estrés cotidiano y afrontamiento. Revista Latinoamericana de Psicología, 42(3), 469-479.

Espejo, T., Chacón, R., Zurita, F., \& Castro, M. (2016). Victimización en edad escolar desde la perspectiva de la actividad física. Sportis, 2(2), 379-389. http://dx.doi.org/10.17979/ sportis.2016.2.3.1729

Estévez, E., Inglés, C. J., \& Martínez-Monteagudo, M. C. (2013). School aggression: Effects of classroom environment, attitude to authority and social reputation among peers. European Journal of Investigation in Health, Psychology and Education, 3(1), 22-32. https://doi.org/10.1989/ejihpe.v3i1.20

Estévez, E., Inglés, C., Emler, N., Martínez-Monteagudo, M. C., \& Torregrosa, M. S. (2012). Análisis de la relación entre la victimización y la violencia escolar: El rol de la reputación antisocial. Intervención Psicosocial, 21, 53-65. http://dx.doi.org/10.5093/ in2012v21n1a3

Estévez, E., \& Jiménez, T. I. (2015). Conducta agresiva y ajuste personal y escolar en una muestra de estudiantes adolescentes españoles. Universitas Psychologica, 14(1), 111-124. https://doi.org/10.11144/Javeriana.upsy14-1.caap

Evans, M., Heriot, S. A., \& Friedman, A. G. (2002). A behavioral pattern of irritability, hostility and inhibited empathy in children. Clinical Child Psychology and Psychiatry, 7(2), 211-224 https://doi.org/10.1177\%2F1359104502007002008

Field, L. (1993). Creating Self-esteem; a Practical Guide to Realizing Your True Worth. Element Books.

Filella-Guiu, G., Pérez-Escoda, N., Agulló, M., \& Oriol, X. (2014). Resultados de la aplicación de un programa de educación emocional en Educación Primaria. Estudios sobre Educación, 26, 125-147. 
Fuentes, M. C., García, J. F., Gracia, E., \& Lila, M. (2011). Autoconcepto y ajuste personal en la adolescencia. Psicothema, 23(1), 7-12.

Gómez, M. C, \& da Resurrección, A. (2017). Estrategias de intervención en conductas disruptivas. Educação por escrito, 8(2), 278-293. http://dx.doi.org/10.15448/21798435.2017.2.27976

Gotzens, C., Badia, M., Castelló, A., \& Genovard, C. (2007). La gravedad de los problemas de comportamiento en el aula vista por los profesores. Revista Portuguesa de Pedagogía 41(1), 103-120. http://dx.doi.org/14195/1647-8614_41-1_7.

Gotzens, C., Badia, M., Genovard, C., \& Dezcallar, T. (2010). Estudio comparativo de la gravedad atribuida a las conductas disruptivas en el aula. Electronic Journal of Research in Educational Psychology, 8(1), 33-58. http://dx.doi.org/10.25115/ ejrep.v8i20.1399

Grebennikov, L. (2005). The Normalised Child: A Non-Traditional Psychological Frame-work. Australian Journal of Early Childhood, 30(2), 8-14. https://doi. org/10.1177/183693910503000204

Gutiérrez-Cobo, M. J., Cabello, R., \& Fernández-Berrocal, P. (2017). Inteligencia emocional, control cognitivo y el estatus socioeconómico de los padres como factores protectores de la conducta agresiva en la niñez y la adolescencia. Revista Interuniversitaria de Formación del Profesorado, 88(31), 39-52. http://hdl.handle. net/10481/49695

Gutiérrez, M., \& Gonçalves, T. O. (2013). Activos para el desarrollo, ajuste escolar y bienestar subjetivo de los adolescentes. International Journal of Psychology and Psychological Therapy, 13(3), 339-355.

Gutiérrez, M. C., \& López, J. E. (2015). Autoconcepto, dificultades interpersonales, habilidades sociales y conductas asertivas en adolescentes. Revista Española de Orientación y Psicopedagogía, 26(2), 42-58. https://doi.org/10.5944/reop.vol.26. num.2.2015.15215

Hampel, P., \& Petermann, F. (2006). Perceived stress, coping, and adjustment in adolescents. Journal of Adolescent Health, 38, 409-415. https://doi.org/10.1016/j. jadohealth.2005.02.014

Heras, J., \& Navarro, R. (2012). Ajuste escolar, soledad y conducta agresiva entre estudiantes de educación secundaria. Revista Qurriculum, 25, 105-124.

Ison, M. (2004). Características familiares y habilidades sociocognitivas en niños con conductas disruptivas. Revista Latinoamericana de Psicología, 36(2), 257-268.

Jenkins, J. M., \& Smith, M. A. (1990). Factors protecting children living in disharmonious homes: maternal reports. American Academy Child Adolescent Psychiatry, 29(1), 6069. https://doi.org/10.1097/00004583-199001000-00011

Kusche, C. A., Greenberg, M. T., \& Beilke, R. (1988). Seattle personality questionnaire for young school-aged children. Unpublished manuscript. University of Washington.

Ladd, G. W., Kochenderfer-Ladd, B., \& Rydell, A. M. (2011). Children's interpersonal skills and school-based relationships. In P. K. Smith y C. H. Hart (Eds.), The Wilwy-Blackwell handbook of childhood social development (pp. 181-206). Wiley-Blackwell. 
Latorre, A., \& Teruel, J. (2009). Protocolo de actuación ante conductas disruptivas. Informació Psicológica, (95), 62-74. http://www.informaciopsicologica.info/OJSmottif/index. php/leonardo/article/view/170

Lazarus, R. S., \& Folkman, S. (1986). Estrés y procesos cognitivos. Martínez Roca.

Lippman, L., \& Rivers, A. (2008). Assessing school engagement: A guide for out-of-school time program practitioners. A Research-to-Results brief. Child Trends.

Martín, M. D. M. B., \& Lleixà, V. G. (2017). Las conductas disruptivas en la escuela rural. Papeles Salmantinos de Educación, (21), 11-33.

Martínez, B., Murgui, S., Musitu, G., \& Monreal, M. C. (2009). El rol de apoyo parental, las actitudes hacia la escuela y la autoestima en la violencia escolar en adolescente. International Journal of Clinical and Health Psychology, 8(3), 679-692.

Martínez, B., Povedano, A., Amador, L.V., \& Moreno, D. (2012). Clima escolar, satisfacción con la vida y victimización en la escuela. Un análisis del efecto moderador del género. Anales de Psicología, 28(3), 875-882. https://www.redalyc.org/articulo. oa?id=16723774026

Matthews, G., Zeidner, M., \& Roberts, R. (2012). Emotional Intelligence 101. Springer.

Méndez, I., \& Cerezo, F. (2010). Bullying y factores de riesgo para la salud en estudiantes de secundaria. European Journal of Education and Psychology, 3(2), 209-210. https://doi.org/10.1989/ejep.v3i2.61

Millon, T. (1998). Trastornos de la personalidad. Más Allá del DSM. Masson.

Mora, I. H., \& Ponce, R. S. (2017). ¿Crece la conflictividad escolar? Percepciones de estudiantes y profesorado de Secundaria. Educatio Siglo XXI, 35(3), 255-275. https://doi.org/10.6018/j/308991

Morente, A. R., Guiu, G. F., Castells, R. R., \& Escoda, N. P. (2017). Análisis de la relación entre competencias emocionales, autoestima, clima de aula, rendimiento académico y nivel de bienestar en educación primaria. Revista Española de Orientación y Psicopedagogía, 28(1), 8-18. http://revistas.uned.es/index.php/reop/article/ view/19355/16270

Murray, J., Farrington, D. P., \& Sekol, I. (2012). Children's antisocial behaviour, mental health, drug use and educational performance after parental incarceration: a systematic review and meta-analysis. Psychological Bulletin, 138(2), 175-210. https://doi. org/10.1037/a0026407

Murray, C., \& Murray, K. M. (2004). Child level correlations of teacher-students relationships: An examination of demographic orientation characteristics, academia orientations and behavioral orientations. Psychology in the Schools, 41(7), 751-762. https://doi. org/10.1002/PITS.20015

Olweus, D., \& Limber, S. (2010). Bullying in School: Evaluation and Dissemination of the Olweus Bullying Prevention Program. American Journal of Orthopsychiatry, 80(1), 124-134. https://psycnet.apa.org/doi/10.1111/j.1939-0025.2010.01015.x

Pérez-Escoda, N., Torrado, M., López-Cassà, E., \& Fernández Arranz, M. (2014). Competencias emocionales y ansiedad en la educación primaria. En: / Congreso Internacional de Educación Emocional (X Jornadas de Educación Emocional): 
"Psicología positiva y bienestar". Grup de Recerca en Orientació Psicopedagògica. Universidad de Barcelona.

Pérez-González, J. C. (2003). Sobre la Validación de Constructo de la Inteligencia Emocional. Encuentros en Psicología Social, 1(2), 252-257.

Porcel, A. M. (2010). Conductas disruptivas en el aula. Revista Digital Innovación y Experiencia Educativa, 34, 1-10.

Porter, R. B., \& Cattell, R. B. (2016). Cuestionario de personalidad para niños. TEA Ediciones.

Ramírez, S., \& Justicia, F. (2006). El maltrato entre escolares y otras conducta-problema para la convivencia. Electronic Journal of Research in Educational Psychology, 4(2), 265290. http://dx.doi.org/10.25115/ejrep.v4i9.1190

Ramos, R., Giménez, A. I., Muñoz-Adell, M. A., \& Lapaz, E. (2006). Cuestionario para la Evaluación de la Autoestima en Educación Primaria. TEA Ediciones.

Reijntjes, A., Stegge, H., Meerum-Terwogt, M., Kamphuis, J. H., \& Telch, M. J. (2006). Children's coping with in vivo peer rejection: An experimental investigation. Journal of Abnormal Child Psychology, 34, 877-889. http://dx.doi.org/10.1007/ s10802-006-9061-8.

Robichaud, M. G. R. (2003). Lénfant Souffre-douleur. Les Editions de L’homme.

Rodríguez, P. L. (2011). Análisis de la convivencia escolar en aulas de educación primaria. Revista Iberoamericana de Educación, 55(3), 1-12. https://doi.org/10.35362/ rie5531599

Rodríguez-Fernández, A., Droguett, L., \& Revuelta, L. (2012). Ajuste escolar y personal en la adolescencia: El papel del autoconcepto académico y del apoyo social percibido. Revista de Psicodidáctica, 17(2), 397-413. http://dx.doi.org/10.1387/Rev. Psicodidact.3002

Romero, N. A. R., Guajardo, J. G., \& Nava, J. M. F. (2017). Competencias socioemocionales como predictoras de conductas prosociales y clima escolar positivo en adolescentes. Revista Interuniversitaria de Formación del Profesorado, 88, 77-90.

Ros, I., Goikoetxea, J., Gairín, J., \& Lekue, P. (2012). Implicación del alumnado en la escuela: diferencias interindividuales e intercentros. Revista de Psicodidáctica, 17(2), 291-307. http://dx.doi.org/10.1387/Rev.Psicodidact.4557

Rosenberg, M. (1965). Society and the adolescent self-image. Princeton University Press.

Rudolph, K. D. (2002). Gender differences in emotional response to interpersonal stress during adolescence. Journal of Adolescent Health, 30(4 Suppl), 3-13. http://dx.doi. org/10.1016/s1054-139x(01)00383-4

Sandstrom, M. J. (2004). Pitfalls of the peer world: How children cope with common rejection experiences. Journal of Abnormal Child Psychology, 32(1), 67-81. https:// doi.org/10.1023/B:JACP.0000007581.95080.8b

Schneider, S. K., O’Donnell, L., Stueve, A., \& Coulter, R. W. (2012). Cyberbullying, school bullying, and psychological distress: A regional census of high school students. American Journal of Public Health, 102(1), 171-177. https://dx.doi. org/10.2105\%2FAJPH.2011.300308 
Schoeps, K., Tamarit, A., González, R., \& Montoya-Castilla, I. (2019). Competencias emocionales y autoestima en la adolescencia: impacto sobre el ajuste psicológico. Revista de Psicología Clínica con Niños y Adolescentes, 6(1), 51-56. https://doi. org/10.21134/rpcna.2019.06.1.7

Seiffge-Krenke, I., \& Stemmler, M. (2002). Factors contributing to gender differences in depressive symptoms: A test of three developmental models. Journal of Youth and Adolescence, 37, 405-417. https://doi.org/10.1023/A:1020269918957

Sun, S., Pan, W., \& Wang, L. L. (2010). A comprehensive review of effect size reporting and interpreting practices in academic journals in education and psychology. Journal of Educational Psychology, 102(4), 989. https://doi.org/10.1037/a0019507

Trianes, M. V., Blanca, M. J., Fernández-Baena, F. J., Escobar, M., \& Maldonado, E. F. (2011). Inventario de Estrés Cotidiano Infantil. TEA Ediciones.

Usán, P., \& Bordás, C. (2018). Motivación escolar, inteligencia emocional y rendimiento académico en estudiantes de educación secundaria obligatoria. Actualidades en Psicología, 32(125), 95-112. https://doi.org/10.15517/ap.v32i125.32123

Valdés, A. A., Sánchez-Escobedo, P. A., \& Carlos-Martínez, E. A. (2012). Autoconcepto social y ajuste escolar de estudiantes de educación media con conductas de hostigamiento en la escuela. Educación y Ciencia, Cuarta época, 2(5), 85-96.

Villarreal-González, M. E., Sánchez-Sosa, J. C., Veiga, F. H., \& Del Moral A, G. (2011). Contextos de desarrollo, malestar psicológico, autoestima social y violencia escolar desde una perspectiva de género en adolescentes mexicanos. Psychosocial Intervention, 20(2), 171-181. https://www.redalyc.org/articulo.oa?id=1798/179819285005

Zimmer-Gembeck, M. J., \& Pronk, R. E. (2012). Relation of depression and anxiety to self-and peer-reported relational aggression. Aggressive Behavior, 38(1), 16-30. https://doi. org/10.1002/ab.20416

Zurita, F., Vilches, J. M., Cachón, J., Padial, R., Martínez, A., \& Castro, M. (2015). Violencia escolar en adolescentes: un análisis en función de la actividad física y lugar de residencia habitual. Universitas Psychologica, 14(2), 759-769. https://doi. org/10.11144/Javeriana.upsy14-2.veaa 